\title{
Pharmacologic agents targeting autophagy
}

\author{
Helin Vakifahmetoglu-Norberg, ${ }^{1}$ Hong-guang Xia, ${ }^{2}$ and Junying Yuan ${ }^{2}$ \\ 'Division of Toxicology, Institute of Environmental Medicine, Karolinska Institutet, Stockholm, Sweden. ${ }^{2}$ Department of Cell Biology, Harvard Medical School, Boston, Massachusetts, USA.
}

\begin{abstract}
Autophagy is an important intracellular catabolic mechanism critically involved in regulating tissue homeostasis. The implication of autophagy in human diseases and the need to understand its regulatory mechanisms in mammalian cells have stimulated research efforts that led to the development of high-throughput screening protocols and small-molecule modulators that can activate or inhibit autophagy. Herein we review the current landscape in the development of screening technology as well as the molecules and pharmacologic agents targeting the regulatory mechanisms of autophagy. We also evaluate the potential therapeutic application of these compounds in different human pathologies.
\end{abstract}

\section{Introduction}

Autophagy is a major intracellular catabolic mechanism that directs selected cytoplasmic components and organelles to the lysosome for degradation. Macroautophagy (hereafter referred to as "autophagy") is responsible for the bulk turnover of cytoplasmic material and is a tightly regulated process. Initiation of autophagy involves the formation of an isolation membrane (phagophore), a critical step in the formation of the autophagosome, and is regulated by multiple signaling mechanisms. The phagophore, which sequesters a region of cytoplasm or selected substrates, elongates and eventually matures into an autophagosome, a double-membrane vesicle that is subsequently trafficked to fuse with a lysosome.

In most cells, autophagy occurs at low basal levels but is often induced to confer stress resistance and sustain cellular survival under adverse conditions, as an essential cytoprotective response (1). Mutations in the autophagic machinery components are associated with a number of human disorders (2). Malfunction of autophagy is implicated in pathophysiology such as cardiomyopathies, infectious diseases, Crohn's disease, and neurodegenerative disorders including Alzheimer's, Huntington's, and Parkinson's diseases (3). Overactivation of autophagy has also been suggested to play an important role in promoting cancer cell survival in the tumor microenvironment in vivo and contributing to resistance to chemotherapies and metabolic changes to sustain tumor cell survival under stress and promote metastasis and dormancy (4-6).

Small molecules are important tools for dissecting molecular mechanisms of biologic pathways and for investigating the potential therapeutic strategies in human diseases. The approach of using small-molecule modulators provides pharmacologic methods similar to those of genetic manipulations to selectively inhibit or activate specific pathways and has therefore been termed "chemical genetics" (7). Compared with conventional genetic approaches, selective small-molecule modulators provide the possibility to

Authorship note: Helin Vakifahmetoglu-Norberg and Hong-guang Xia are co-first authors.

Conflict of interest: The authors have declared that no conflict of interest exists. Reference information: J Clin Invest. 2015;125(1):5-13. doi:10.1172/JCI73937. conditionally regulate the activities of gene products in different settings, including cultured cell models and animal models. In contrast to permanent genetic modification, small-molecule tools provide a means to regulate target activity in a temporal and often reversible manner. During the past several years, a variety of smallmolecule modulators that either activate or inhibit autophagy pathways have been developed that will be the subject of this Review.

Due to its pathophysiologic significance, autophagy has been the subject of intensive study, in the effort to gain a better understanding of the process at the molecular level and to discover potential new therapeutic targets. Small-molecule activators of autophagy have been shown to reduce the amounts of toxic protein aggregates and to promote cell survival under stress, which may be of therapeutic benefit in certain neurodegenerative diseases. In addition, the induction of autophagy has been shown to increase the longevity of certain experimental organisms (8), suggesting that the activation of autophagy may help to maintain normal homeostasis during aging and promote longevity. On the other hand, there are also circumstances in which autophagic activation may permit pathogenesis. For example, the inhibition of autophagy as a therapeutic approach might eliminate stress-tolerant cancer cells that escape chemotherapeutic treatment and which currently pose a fundamental barrier to successful anticancer treatments. Accordingly, both pharmacologic activators and inhibitors of autophagy may be considered as potential new drug candidates for the treatment of autophagy-relevant human diseases.

Herein we review the current landscape in the development of screening technology and the discovery of molecules and pharmacologic agents that target various regulatory mechanisms of the autophagic machinery. We highlight recent advances in the discovery of small-molecule regulators of autophagy and evaluate their utility in dissecting autophagic pathways and the potential therapeutic application of these compounds in human pathogenesis.

\section{Screening strategies for isolating autophagy regulators}

The hallmark for activation of autophagy is the formation of double-membraned autophagosomes that can only be clearly discerned at the EM level, which is incompatible with the demand for 


\section{Table 1. Compounds that induce autophagy}

\begin{tabular}{|c|c|c|}
\hline Compound & Mechanism and use & Reference \\
\hline Rapamycin & Inhibits mTORC1 & 17,18 \\
\hline Temsirolimus/CCI-779 & Inhibits mTORC1; approved for the treatment of advanced renal cell carcinoma & 19 \\
\hline Everolimus & Inhibits mTORC1; approved for cancer treatment and as an immunosuppressant for organ transplant recipients & 20 \\
\hline PP242 & ATP-competitive inhibitor of mTORC1/2 & 26,28 \\
\hline Torin 1 & Selective ATP-competitive inhibitor of mTORC1/2 & 27 \\
\hline GDC-0980 & Inhibits class I PI3K and mTORC1/2 & 48 \\
\hline GDC-0941 & Inhibits class I PI3K & 49 \\
\hline Perifosine & Inhibits AKT & 50 \\
\hline Fluspirilene & $\begin{array}{l}\text { Reduces intracellular } \mathrm{Ca}^{2+} / \mathrm{calpain} 1 \text { activity and induces autophagy } \\
\text { by preventing calpain 1-mediated cleavage of ATC5; used as an antipsychotic }\end{array}$ & 10,54 \\
\hline Pimozide & Mechanism unknown; used as an antipsychotic & 10 \\
\hline L-690330 & Inhibits IMPase & 58 \\
\hline Carbamazepine & Reduces intracellular inositol levels; used as an anticonvulsant and mood stabilizer & 58 \\
\hline Xestospongin B & Competitive inhibitor of IP3Rs & 59 \\
\hline Minoxidil & Mechanism unknown; used as an antihypertensive vasodilator & 15 \\
\hline Clonidine & Mechanism unknown; used as an $\alpha_{2}$-adrenergic agonist & 15 \\
\hline Verapamil & $\mathrm{Ca}^{2+}$ channel antagonist; used to treat hypertension and cardiac arrhythmia & 10,15 \\
\hline Loperamide & Mechanism unknown; opioid receptor agonist used to treat diarrhea & 10,15 \\
\hline Amiodarone & Mechanism unknown; used to treat cardiac arrhythmia & 10,15 \\
\hline Nimodipine & $\mathrm{Ca}^{2+}$ channel antagonist; used to treat hypertension & 15 \\
\hline Nitrendipine & $\mathrm{Ca}^{2+}$ channel antagonist; used to treat hypertension & 15 \\
\hline Rilmenidine & $\begin{array}{l}\text { Imidazoline receptor } 1 \text { agonist; used to treat hypertension, and safety } \\
\text { as autophagy inducer currently being tested in patients with Huntington's disease }\end{array}$ & 61 \\
\hline Noscapine & Benzylisoquinoline alkaloid that activates autophagy and inhibits IL-1 $\beta$ production; used as a cough suppressant & 12 \\
\hline Clemastine & Activates autophagy and inhibits IL-1 $1 \beta$ production; used as an antihistamine and anticholinergic drug & 12 \\
\hline Tat-beclin 1 peptide & Releases beclin 1 from Golgi into cytoplasm to mediate the formation of autophagosomes, and promotes autophagic cell death & 62,63 \\
\hline
\end{tabular}

a high-throughput screening approach. Fortunately, the identification of GFP-tagged light chain 3 (GFP-LC3), one of the mammalian homologs of Atg8 in yeast that targets the isolation membrane upon the formation of autophagosomes as a fluorescent marker for autophagy (9), and the recent development of high-throughput microscopy technology made it possible to conduct such image-based screens using light microscopy. In fact, image-based screens for small-molecule modulators of autophagy represent a prominent example of the successful use of image-based, highthroughput screens by multiple labs.

GFP-LC3-II-based methods. GFP-LC3 was developed as an image-based method to quantitate autophagosomes as ringshaped structures or small dots in the cytoplasm upon induction of autophagy (9). Zhang et al. adapted this method into a highthroughput imaging-based screening system using human glioblastoma H4 cells stably expressing human GFP-LC3 (10). The level of autophagy was analyzed by measuring the number, size, and intensity of GFP-LC3 spots using high-throughput fluorescence microscopy. Treatment with rapamycin, a known autophagy inducer, leads to significant increases in the fluorescence levels of GFP-LC3 compared with DMSO-treated cells. However, since the induction of autophagy is commonly associated with activation of cellular stress and cell death, which can be very common when screening a small-molecule library, the hit rate of autophagy inducers can be very high. To eliminate those compounds that induce autophagy as a consequence of cell death, it is important 


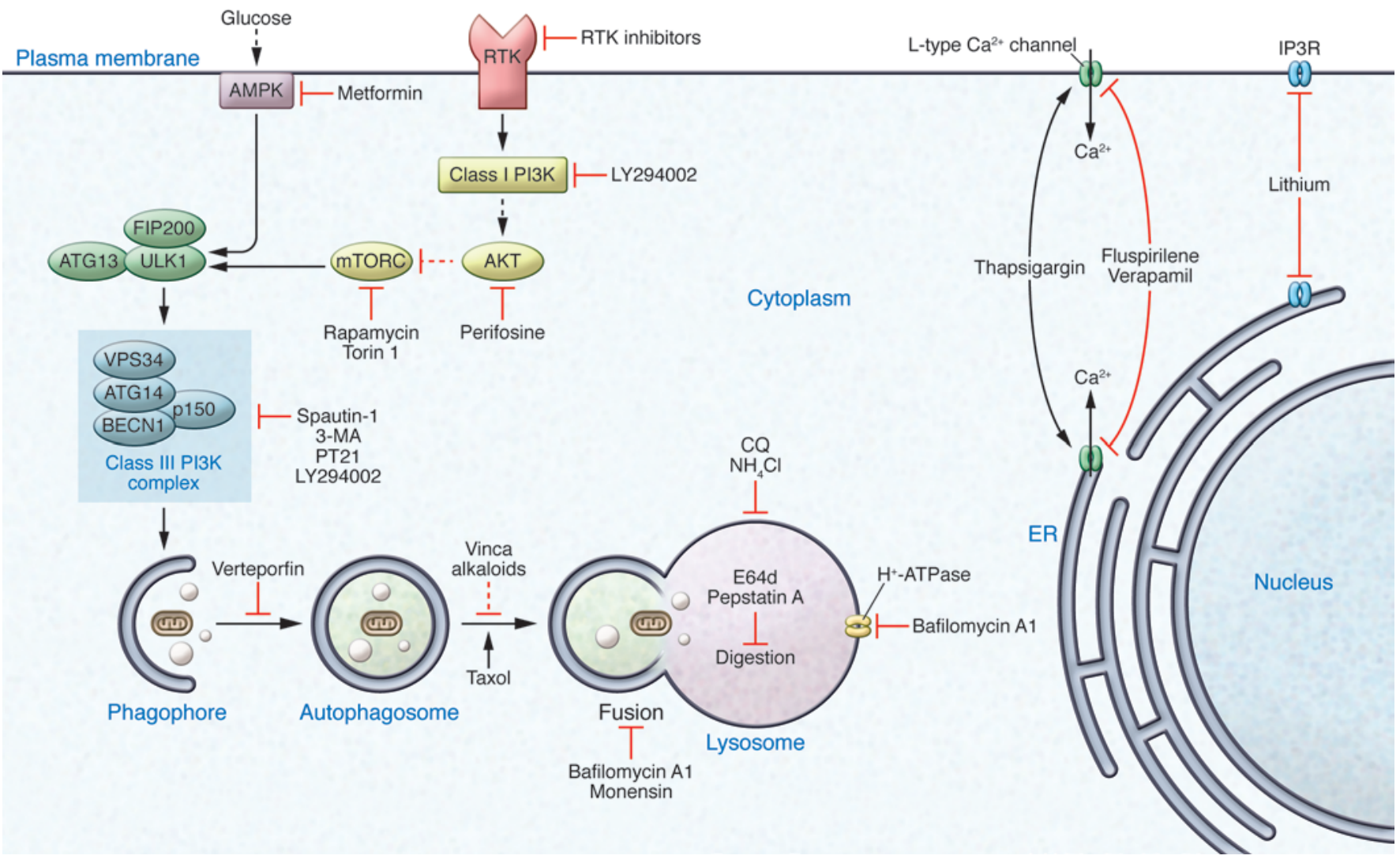

Figure 1. Small-molecule modulators of autophagy that target different steps of the autophagic machinery. Autophagy can be activated by inhibitors against class I PI3K (LY294002), mTOR (rapamycin), AKT (perifosine), and IMPase (Li+) as well as activation of AMPK by metformin. While Ca ${ }^{2+}$ channel inhibitors (fluspirilene, verapamil) can stimulate autophagy, thapsigargin, an endoplasmic reticulum $\mathrm{Ca}^{2+} \mathrm{ATPase}$ inhibitor, elevates Ca ${ }^{2+}$ and suppress autophagy. The initiation of autophagy is regulated by the activity of the class III PI3K complex, which can be inhibited by 3-MA and PT21 as well as by spautin-1 that can promote the degradation of the class III PI3K complexes. Once the phagophore formation is initiated, compounds such as verteporfin can interfere with LC3-interacting region motifs and potentially block the selective recruitment of cargos such as mitochondria. The trafficking of autophagosomes to the lysosome is facilitated by the cytoskeleton. Thus, microtubule destabilization by vinca alkaloids can block the maturation of autophagosomes, whereas stabilization by taxol may increase the fusion between autophagic vacuoles and lysosomes. On the other hand, paclitaxel was shown to inhibit autophagy by blocking the activation of the VPS34 complex by inducing inhibitory phosphorylation of VPS34 as a substrate of CDK1. Lysosomotropic agents that increase the lysosomal $\mathrm{pH}$, such as $\mathrm{CQ}, \mathrm{NH}_{4} \mathrm{Cl}$, and monensin, interfere with lysosomal function and block autophagy at a late stage. The final step of the autophagy pathway can also be blocked by inhibitors of lysosomal enzymes, such as E64d and pepstatin A, and by bafilomycin A1, a specific inhibitor of v-ATPase.

to include secondary criteria in the screens, e.g., by determining the effects of the compounds on cell viability at the same time. To test whether the activation of autophagy might accelerate the degradation of misfolded proteins, Zhang et al. used the accumulation of mutant polyglutamine as another marker, which can be degraded upon the activation of autophagy. With this screening method, both autophagy activators and inhibitors were isolated successfully $(10,11)$.

Shaw et al. used a dual fluorescent reporter mCherry-GFP-LC3, which can potentially distinguish between increases in $\mathrm{GFP}^{+}$punctae as a result of increased autophagic flux and impaired lysosomal function due to the ability of acidic lysosomal pH to quench the GFP signal but not the mCherry signal (12). Since defects in key autophagy genes such as Atg16l1 have been shown to lead to increased intestinal inflammation (13), Shaw et al. tested the possibility of isolating autophagy inducers that can also block the production of the inflammatory mediator IL-1 $1 \beta$. This screen was able to identify compounds that can induce autophagy as well as block IL-1ß production and demonstrated the feasibility of identifying small molecules that can both induce autophagy and block inflammation.

p62/SQSTM1-based methods. Another imaging-based screening method for autophagy regulators involves the measurements of p62, an autophagy receptor that functions by linking autophagic cargo to ATG8/LC3 family members located on the autophagic membranes. Larsen et al. set up a cell system based on tetracycline-induced expression of GFP-p62 using Flp-In T-REx HEK293 cells, with GFP-p62 recombined into a single integrated FRT site in the genome (14). This system allows for the monitoring of inducible autophagic flux by following the degradation of the fusion proteins after promoter shut-off. This inducible GFP-p62 system does not require the quantification of autophagosome size or intensity and thus may be more straightforward for adaption by other laboratories.

Mutant protein-based methods. Williams et al. developed a method based on the detection of removal of hemagglutinintagged mutant $\alpha$-synuclein or GFP-fused mutant huntingtin pro- 


\section{Table 2. Compounds that inhibit autophagy}

\begin{tabular}{|c|c|c|}
\hline Compound & Mechanism and use & Reference \\
\hline 3-MA & Inhibits class III PI3K & 38 \\
\hline Wortmannin/LY294002 & Inhibits class III PI3K & 41 \\
\hline PT21 & Inhibits VPS34 kinase & 38 \\
\hline Spautin-1 & Inhibits USP10/13 to promote the degradation of VPS34 complexes & 11,45 \\
\hline Thapsigargin & A non-competitive inhibitor of SERCA that blocks the fusion of autophagosomes with lysosomes & 56 \\
\hline Paclitaxel & $\begin{array}{c}\text { Microtubule stabilizer that inhibits autophagy by inducing inhibitory phosphorylation of VPS34 at T159 and blocking } \\
\text { autophagosome-lysosome fusion }\end{array}$ & 68,69 \\
\hline CQ & $\begin{array}{c}\text { Neutralizes the acidic pH of intracellular vesicles and inhibits autophagy by blocking lysosomal degradation; } \\
\text { used to treat and prevent malaria }\end{array}$ & 77 \\
\hline HCQ & A CQ derivative that blocks lysosomal degradation & 78 \\
\hline Lys05 & A CQ derivative with improved lysosomal accumulation & 79 \\
\hline Monensin & Interferes with autophagosome-lysosome fusion; used as an antibiotic & 81 \\
\hline Lucanthone & Interferes with lysosomal degradation; used as a chemotherapeutic agent and DNA intercalator & 82 \\
\hline Azithromycin & Inhibits the activity of v-ATPases and blocks autophagosome-lysosome fusion; used as an antibiotic & 89 \\
\hline E64d/Pepstatin A & Inhibitors of cysteine and aspartic proteases that inhibit autophagy by blocking lysosomal degradation & 90 \\
\hline Bafilomycin A1 & Inhibitor of v-ATPase that blocks the lysosomal proton transport, leading to the inhibition of lysosomal hydrolases & 87 \\
\hline Concanamycin A & Inhibitor of v-ATPase that blocks the lysosomal proton transport and leads to the inhibition of lysosomal hydrolases & 88 \\
\hline Verteporfin & Used in combination with photodynamic therapy to eliminate abnormal blood vessels & 91 \\
\hline DCMI & Inhibits autophagosome-lysosome fusion; used as an antidepressant & 92 \\
\hline
\end{tabular}

teins, both of which are aggregate prone and are known autophagy substrates (15). The expression levels of these mutant proteins in cells treated with small molecules are measured using antibodies against hemagglutinin or GFP as a surrogate marker for mutant protein levels.

\section{Pharmacological agents that target autophagic machinery}

Here we provide a discussion of small-molecule activators and inhibitors of autophagy that have been used in research and their potential application in developing new treatments for human diseases (Figure 1 and Tables 1 And 2). We caution the reader that since we are still in the early days of developing small-molecule modulators of autophagy, we know of few if any small molecules that can directly target the autophagy machinery, and most compounds discussed here affect the regulatory mechanisms of autophagy. Their efficacy in modulating autophagy has not been thoroughly investigated in humans. Even for those FDA-approved drugs that have been shown in experimental systems to modulate autophagy, it remains to be tested whether the concentrations required to modulate autophagy in vivo can be safely achieved. On the other hand, the identification and development of small-molecule modulators of autophagy have been important and useful for investigating molecular mechanisms and potential indications for autophagy regulation in the treatment of human diseases.

Inhibitors of mTOR signaling activate autophagy. mTOR, a member of the PI3K family, is an important regulator of multiple intracellular processes that include protein translation as well as autophagy and metabolism in response to different nutrient, energy, and growth factor-signaling conditions (16). mTOR is the catalytic subunit of two functionally distinct complexes, mTOR complex 1 (mTORC1) and mTORC2. mTORC1 inhibitors, including rapamycin or rapalogs, can affect the activity of Unc-51 like autophagy activating kinase 1 (ULK1), a key Ser/Thr kinase that acts both as a downstream effector and negative regulator of mTORC1 to regulate autophagy (17-21). Activation of autophagy by rapamycin and its analogs has been shown to be protective in different animal models of neurodegenerative disorders (22-25). These data suggest that autophagy prevents the accumulation of misfolded proteins. A new generation of ATP-competitive inhibitors of mTOR display potent activation of autophagy. Unlike that of rapamycin, which only inhibits mTORC1, this new generation of inhibitors, such as PP242 and Torin 1, inhibits both mTORC1 and mTORC2 (26-29).

Although rapamycin and other inhibitors of mTOR are strong activators of autophagy and have been tested as anticancer therapeutics, caution should be taken in equating the activation of autophagy with cancer suppression, as accumulating evidence suggests that activation of autophagy can promote the survival of cancer cells. By promoting intracellular recycling, autophagy can confer stress resistance and sustain cell survival under adverse conditions, which may promote metastasis (30). Thus, the efficacy of rapamycin and other inhibitors of $\mathrm{mTOR}$ as an anticancer therapy may have to do with their inhibitory effects on protein translation and metabolism, rather than autophagy activation.

Activators of AMPK activate autophagy. AMPK, the main sensor of intracellular energy, is an important regulator of both ULK1 and mTOR. The activation of AMPK by increases in the intracellular ratio of AMPK/ATP leads to the activation of autophagy via both inhibition of mTOR and activation of ULK1 
(31). Hence the administration of AMPK activators such as metformin stimulates autophagy. The mechanism of metformin is still being debated: metformin has been reported to target the LKB1/AMPK complexes (32) and/or inhibit the respiratory chain complex I, which leads to a reduction in intracellullar ATP, thereby indirectly activating AMPK (33).

Inhibitors of VPS34 kinase complexes block autophagy. Recent evidence suggests that modulation of the activity of class III PI3Ks, including VPS34, PIK3R4, ATG14L/Barkor, UVRAG, BIF-1, Rubicon, and Ambra1 (34-36), regulates autophagy. Class III PI3K complexes mediate the production of phosphatidylinositol3-phosphate (PI3P), a key lipid-signaling molecule that serves as an important checkpoint involved in regulating the mTOR-independent induction of autophagy under normal nutritional conditions (37). PI3P is required for the formation of the phagophore and, subsequently, the autophagosome.

Autophagy is positively regulated by PI3P, the product of class III PI3K (VPS34 complexes), and is negatively regulated by phosphatidylinositol triphosphate, the production of class I PI3K. 3-Methyladenine (3-MA) has been widely used as an inhibitor of class III PI3K to block autophagosome formation; however, 10 mM 3-MA is required to inhibit autophagy. A structural study suggested that 3-MA preferentially inhibits VPS34 in vitro (38). In vivo, $10 \mathrm{mM}$ 3-MA can also inhibit class I PI3K, which may explain why it can promote autophagy flux under nutrient-rich conditions $(39,40)$. The PI3K inhibitors wortmannin and LY294002, which inhibit both class I and III PI3K complexes, have also been used to inhibit autophagy $(39,41,42)$.

An x-ray crystallographic study of the class III PI3K VPS34 demonstrated that it exhibits distinguishing structural features that differentiate it from class I PI3Ks. The P loop of VPS34, which binds the phosphates of ATP, curls inward toward the ATPbinding pocket and is more constricted than that of class I PI3K. The hinge between the N and C lobes of VPS34 is one residue shorter than in class I PI3Ks. Therefore, VPS34 lacks the bulgedout space at the adenine-binding pocket hinge that is characteristic of class I PI3Ks. Miller et al. exploited these structural characteristics of VPS34 and designed a number of promising VPS34 kinase inhibitors such as PT21 ( $\left.\mathrm{IC}_{50} 88 \mathrm{nM}\right)$ (38). Further development of these VPS34 kinase inhibitors may provide promising leads to target the class III PI3K.

The stability of VPS34 complex components, including VPS34, beclin 1, VPS15/p150, ATG14L, and UVRAG, are coordinately regulated so that the reduction of one component may lead to the reduction in the levels of other components (43). Thus, it is possible to regulate the levels of entire complexes by targeting one component. Spautin-1, isolated from an imaged-based screen for small-molecule modulators of autophagy (11), promotes the degradation of VPS34 complexes by targeting the ubiquitin-specific peptidases, USP10 and USP13, two deubiquitinating enzymes that regulate beclin 1 and VPS34 stability. Spautin-1 promotes ubiquitination and proteasomal degradation of beclin 1 and VPS34 complexes under glucose-free conditions (44), consequently inhibiting autophagy.

Spautin-1 treatment can promote the death of cancer cells in the setting of nutrient deprivation when autophagy is activated to promote survival. Moreover, inhibition of autophagy by spautin- 1 under nutritional deprivation conditions leads to the activation of chaperone-mediated autophagy, a lysosome-dependent pathway that mediates degradation of missense mutant p53 protein (45). Increasing evidence suggests that certain p53 mutants may promote oncogenesis through a dominant gain-of-function mechanism; thus, the reduction of accumulated mutant p53 may be an important therapeutic goal in cancer treatment. Development of improved spautin-1 analogs with good safety and in vivo stability profiles may represent a novel anticancer therapeutic strategy.

Inhibitors of class I PI3K signaling activate autophagy. In contrast to the positive role of the class III PI3K products in promoting autophagy, increases in the levels of class I PI3K products (phosphatidylinositol 3,4-bisphosphate and phosphatidylinositol 3,4,5-triphosphate) negatively regulate autophagy (46). Targeting this class I PI3K with inhibitors such as CH5132799, GDC-0980, and GDC-0941, leads to the activation of autophagy (47-49).

Protein kinase AKT is a major downstream mediator of class I PI3K signaling. Pharmacologic targeting of AKT by inhibitors such as perifosine leads to autophagy activation (50). Moreover, treatment with inhibitors of receptor tyrosine kinases that leads to the inhibition of AKT can activate autophagy, possibly by inhibiting mTORC1 or by regulating beclin 1 . The activation of the EGFR tyrosine kinase-mediated signaling pathway may inhibit autophagy through multiple mechanisms that target beclin $1(51,52)$.

Modulators of intracellular $\mathrm{Ca}^{2+}$ affect autophagy. $\mathrm{Ca}^{2+}$ is an important intracellular second messenger involved in the regulation of many cellular processes, including autophagy, which can be inhibited by increasing intracytosolic $\mathrm{Ca}^{2+}$ levels (53). L-type $\mathrm{Ca}^{2+}$ channels are voltage-dependent channels that are involved in regulating excitation-contraction coupling of muscle and hormone secretion in endocrine cells. Several L-type $\mathrm{Ca}^{2+}$ channel antagonists were isolated from multiple screens for small-molecule modulators of autophagy. Zhang et al. (10) identified multiple antagonists of L-type $\mathrm{Ca}^{2+}$ channels, including fluspirilene, verapamil, loperamide, and amiodarone, as autophagy activators that promote the degradation of long-lived proteins and misfolded polyglutamine but do not cause cell death. In another small-molecule screen for autophagy modulators using the clearance of the autophagy substrate A30P $\alpha$-synuclein as a biomarker, Williams et al. (15) also uncovered multiple antagonists of $\mathrm{L}$-type $\mathrm{Ca}^{2+}$ channels including verapamil, loperamide, amiodarone, nimodipine, and nitrendipine as autophagy inducers that do not exhibit cytotoxicity.

The ability of these L-type $\mathrm{Ca}^{2+}$ channel antagonists to induce autophagy suggests the possibility that the levels of intracellular $\mathrm{Ca}^{2+}$ play an important role in regulating autophagy. Elevation of intracellular $\mathrm{Ca}^{2+}$ leads to the activation of calpains, which are $\mathrm{Ca}^{2+}$-dependent, non-lysosomal cysteine proteases that are expressed ubiquitously in mammals and other organisms. The treatment of fluspirilene, an antipsychotic drug used for the treatment of schizophrenia, was found to reduce intracellular $\mathrm{Ca}^{2+}$ and prevent the calpain-mediated cleavage of ATG5, which is activated by elevated intracellular $\mathrm{Ca}^{2+}(54)$. In addition, calpain 1 can cleave the $G$ as subunit of heterotrimeric $G$ proteins, which increases its activity after calpain cleavage. G $\alpha s_{\alpha}$ enhances adenylyl cyclase activity, which in turn inhibits autophagy (55). Thus, a reduction in the intracellular $\mathrm{Ca}^{2+}$ concentrations and calpain activity can lead 
to the activation of autophagy. These studies suggest that calpain 1 may have multiple mechanisms to control the levels of autophagy in cells under normal nutritional conditions by regulating the levels of key proteins involved in autophagy.

Thapsigargin, a noncompetitive sarcoplasmic reticulum/ER $\mathrm{Ca}^{2+}$ ATPase inhibitor, increases cytosolic $\mathrm{Ca}^{2+}$ concentrations by inhibiting $\mathrm{Ca}^{2+}$ pumps in the sarcoplasmic reticulum and ER. Depletion of $\mathrm{Ca}^{2+}$ in the ER may in turn cause secondary activation of plasma membrane $\mathrm{Ca}^{2+}$ channels, allowing further $\mathrm{Ca}^{2+}$ influx into the cytosol. Thapsigargin does not affect autophagosome formation but leads to accumulation of mature autophagosomes by specifically inhibiting the fusion of autophagosomes with lysosomes (56). Thapsigargin blocks autophagosomal recruitment of the small GTPase RAB7, which is required for complete autophagic flux. However, disruption of $\mathrm{Ca}^{2+}$ homeostasis by thapsigargin also leads to ER stress, which could induce autophagy (53). Another study demonstrates that thapsigargin causes accumulation of mature autophagosomes by blocking autophagosome fusion within the endocytic system (56). Thus, while the activation of ER stress can induce autophagy, disruption of intracellular $\mathrm{Ca}^{2+}$ homeostasis can inhibit autophagy.

Inhibitors of inositol monophosphatase activate autophagy. Lithium, used widely as a mood-stabilizing drug for the treatment of bipolar disorder, was shown to induce autophagy and reduce mutant huntingtin aggregates and cell death (57). Sarkar et al. found that lithium inhibits inositol monophosphatase (IMPase), leading to depletion of free inositol and a reduction in the levels of myo-inositol-1,4,5-triphosphate (IP3), which may represent an mTOR-independent pathway for regulating mammalian autophagy. Similar effects were observed with other IMPase inhibitors and mood-stabilizing drugs such as L-690330 and carbamazepine, which also decrease inositol levels (58). Since IP3 receptor (IP3R) is a potential binding partner of beclin 1, the IP3R antagonist Xestospongin B can stimulate autophagy by releasing beclin 1 from IP3R to assemble into the VPS34 complex $(59,60)$. In addition, rilmenidine, which is an imidazoline receptor 1 agonist and an FDA-approved drug for hypertension, has been shown to induce autophagy, enhance mutant huntingtin clearance and reduce toxicity in a mouse model of Huntington's disease. Rilmenidine is currently being tested in a safety trial in patients with Huntington's disease (61). Any success of such clinical trials would encourage the development of additional autophagy modulators for the treatment of neurodegenerative diseases.

Induction of cell death by Tat-beclin 1 peptide. Tat-beclin 1 peptide, a fusion peptide of 18 amino acids derived from beclin 1 and the HIV Tat protein transduction domain, was shown to induce autophagy and decrease the accumulation of polyglutamine expansion protein aggregates by binding to Golgi-associated plant pathogenesis-related protein 1 (GAPR-1, also known as GLIPR2), a protein that associates with lipid rafts at the cytosolic leaflet of the Golgi membrane. In addition, Tat-beclin 1 inhibits the replication of several pathogens (including HIV-1) in vitro and reduces mortality in mice infected with chikungunya or West Nile virus (62). On the other hand, treatment with Tat-beclin 1 can induce cell death (63), and knockdown of components of the autophagy machinery, such as beclin 1, Atg14L, and Atg5, can block cell death induced by Tat-beclin 1, which suggests that Tat-beclin 1 may activate autophagic cell death.
Targeting cytoskeletal components to regulate autophagy. The destabilization of microtubules by either vinblastine (64), nocodazole, or cytochalasin B and D blocks the maturation of autophagosomes (65); conversely, taxol-mediated microtubule stabilization increases the fusion between autophagic vacuoles and lysosomes $(66,67)$. Paclitaxel, which also stabilizes microtubules, was shown to inhibit autophagy through two distinct mechanisms dependent on the cell cycle stage. In mitotic cells, paclitaxel blocks the activation of the VPS34 complex by inducing inhibitory phosphorylation of VPS34 at T159, which is mediated by mitotic kinases such as CDK1 (68). In non-mitotic cells, paclitaxel inhibits autophagosome trafficking to block maturation of autophagic vesicles (69). The ability of microtubule targeting agents to negatively regulate autophagy suggests that their efficacy as anticancer therapeutics may be partially attributable to their ability to inhibit autophagy. Additionally, other autophagy-inhibiting agents may not enhance the therapeutic effects of paclitaxel, nocodazole, or vinblastine, as these agents already inhibit autophagy.

Histone deacetylase 6 (HDAC6), a microtubule-associated deacetylase, has been shown to function as a central component of basal autophagy that targets protein aggregates to the dynein motor for transport $(70,71)$. HDAC6 was shown to promote autophagy by recruiting cortactin-dependent, actin-remodeling machinery, which assembles an F-actin network that stimulates autophagosome-lysosome fusion and substrate degradation (72). HDAC6 deficiency impairs autophagosome maturation; accordingly, inhibitors of HDAC6 and other HDACs have been show to block autophagy (73) through acetylation and suppression of autophagy-associated proteins such as ATG7. However, the effect of HDAC inhibitors on autophagy has been a controversial issue, and other studies propose HDAC inhibitors as autophagy activators (74-76).

Lysosomal alkalizers block autophagic flux and degradation. The lysosomal lumen alkalizers inhibit autophagy by neutralizing the acidic $\mathrm{pH}$ in the lumen of lysosomal vesicles, which is required for the activities of lysosomal hydrolases involved in autophagic degradation. Thus, alkylation of lysosomal vesicles leads to the accumulation of autophagosomes by blocking lysosomal degradation (77). Chloroquine (CQ) and its derivative hydroxychloroquine (HCQ) have been investigated in preclinical studies and clinical trials as anticancer drug candidates (78). Some efforts have been made to generate more potent inhibitors of lysosomal functions. For example, Lys05, a dimeric CQ with an improved ability to accumulate in the lysosomes, may have more potent antitumor activity than HCQ $(79,80)$. Other compounds with CQ-like activities in alkalizing lysosomal compartments have also been identified. Monensin, a polyether antibiotic, is also a lysosomotropic drug that prevents the acidification of lysosomes and interferes with the fusion of autophagosomes and lysosomes (81). In addition, lucanthone, an anti-schistosome agent, inhibits autophagy via a similar mechanism to CQ (82). Finally, matrine, a quinolizidine alkaloid, can also block autophagic degradation by elevating the luminal $\mathrm{pH}$ of lysosomes (83).

AAA ATPase inhibitors block autophagic degradation. p97 (also called valosin containing protein [VCP]) is implicated in autophagosome maturation (84) under basal conditions and in cells treated by proteasome inhibition, but not in cells challenged by 
starvation, suggesting that p97 might be selectively required for autophagic degradation of ubiquitinated substrates. Xanthohumol, a prenylated chalcone that can bind directly to the $\mathrm{N}$ domain of p97, was identified as a p97 ATPase inhibitor that can modulate autophagy (85). DBeQ, a selective, reversible and ATP-competitive p97 inhibitor, also impairs autophagic degradation of LC3-II and proteasomal degradation of a p97-dependent ubiquitin-proteasome system (86). Because inhibiting the ATPase activity of p97 by $\mathrm{DBeQ}$ negatively regulates both autophagy and proteasomemediated degradation, the two key catabolic mechanisms of cells, DBeQ effectively induces caspase activation and apoptosis.

Vacuolar $\mathrm{H}^{+}$-ATPase inhibitors block autophagic flux. Vacuolar $\mathrm{H}^{+}$-ATPases (v-ATPases) on the membrane of intracellular organelles with acidic lumen, such as endosomes and lysosomes, couple proton transport with ATP hydrolysis to maintain the proton gradient and the acidic $\mathrm{pH}$ environment. Treatment of inhibitors of v-ATPases, such as bafilomycin A1 or concanamycin A $(87,88)$, block lysosomal proton transport and lead to the inhibition of lysosomal hydrolases, which are only activated at a low $\mathrm{pH}$. As a consequence, inhibition of v-ATPase blocks autophagic flux.

Azithromycin, a macrolide antibiotic drug commonly used to treat certain bacterial infections, has been identified as an autophagy inhibitor by preventing lysosomal acidification (89). Inhibition of autophagy by azithromycin has been suggested to lead to inhibition of intracellular killing of mycobacteria within macrophages and result in chronic infection with non-tuberculous mycobacteria in patients with chronic inflammatory lung diseases such as cystic fibrosis, who may require long-term use of antibiotics such as azithromycin.

Other inhibitors of autophagic degradation. Inhibitors of lysosomal hydrolases and proteases, such as E64d and pepstatin A, are commonly used to target cysteine, serine, and threonine peptidases such as leupeptin or cystatin B, or cathepsins that are localized in the lysosomal lumens to block autophagic flux (90).

Verteporfin, a benzoporphyrin derivative and an FDA-approved drug used clinically in photodynamic therapy, was identified in an image-based screen for chemicals that can prevent autophagosome formation in the presence of the lysosomal inhibitor CQ (91). Since the cytoplasm of cells treated with verteporfin exhibits the accumulation of single-membrane vesicles that are empty by EM analysis, it was hypothesized that verteporfin might inhibit the membrane expansion of phagophores, leading to defects in capturing cytoplasmic cargo.

Clomipramine, an FDA-approved drug used for the treatment of psychiatric disorders, and its active metabolite, desmethylclo- mipramine (DCMI), have been shown to interfere with autophagic flux by blocking autophagosome-lysosome fusion (92). DCMI-dependent blockage of autophagy enhances the efficacy of doxorubicin, a chemotherapeutic agent (92), but further studies are needed to clarify its anti-autophagic mechanism.

\section{Concluding perspectives and future directions}

Since defects in autophagic pathways have been implicated in many human disorders from inflammatory diseases to cancers and neurodegeneration, augmentation or inhibition of autophagy could potentially be used to treat multiple human diseases. While we are still in the early days in terms of developing pharmacologic modulators of autophagy, the identification of small-molecule modulators has already provided important insights into the mechanisms that regulate autophagy. High-throughput, imagebased screens have been developed by multiple labs for isolating small-molecule modulators of autophagy. This was made possible by the recent technology development on fluorescence microscopy and related software platforms that allow quantitative analysis of autophagic vesicles in fluorescent imagery. The combination of high-throughput, image-based screens and additional criteria such as cell viability and production of inflammatory cytokines has made it possible to identify small-molecule modulators of autophagy with certain specificities. With the advancement in our understanding of autophagy pathways, we expect that there will be more target-specific design of autophagy modulators, such as inhibitors of VPS34 kinase activity. Small molecules that interfere with the various steps of the autophagic machinery that act as inducers or inhibitors will provide important tools for understanding the mechanism of autophagy and provide potential lead candidates for developing novel therapeutics for human diseases.

\section{Acknowledgments}

This work was supported in part by grants to J. Yuan from the National Institute of Neurological Disorders and Stroke (1R01NS082257) and the National Institute on Aging (R37 AG012859) and a Senior Fellowship from the Ellison Foundation. This work was also supported by grants to H. VakifahmetogluNorberg from the Ake Wibergs Foundation and The Lars Hiertas Memorial Foundation.

Address correspondence to: Junying Yuan, Department of Cell Biology, Harvard Medical School, 240 Longwood Avenue, Boston, Massachusetts 02115, USA. Phone: 617.432.4170; E-mail: jyuan@ hms.harvard.edu.
1. Moreau K, Luo S, Rubinsztein DC. Cytoprotective roles for autophagy. Curr Opin Cell Biol. 2010;22(2):206-211.

2. Mizushima N, Levine B, Cuervo AM, Klionsky DJ. Autophagy fights disease through cellular selfdigestion. Nature. 2008;451(7182):1069-1075.

3. Nixon RA. The role of autophagy in neurodegenerative disease. Nat Med.2013;19(8):983-997.

4. Yue Z, Jin S, Yang C, Levine AJ, Heintz N. Beclin 1, an autophagy gene essential for early embryonic development, is a haploinsufficient tumor suppressor. Proc Natl Acad Sci US A.
2003;100(25):15077-15082.

5 . Qu X, et al. Promotion of tumorigenesis by heterozygous disruption of the beclin 1 autophagy gene. J Clin Invest. 2003;112(12):1809-1820.

6. Lock R, et al. Autophagy facilitates glycolysis during Ras-mediated oncogenic transformation. Mol Biol Cell. 2010;22(2):165-178.

7. Schreiber SL. Chemical genetics resulting from a passion for synthetic organic chemistry. Bioorg Med Chem. 1998;6(8):1127-1152.

8. Rubinsztein DC, Mariño G, Kroemer G. Autophagy and aging. Cell. 2011;146(5):682-695.
9. Mizushima N, Yamamoto A, Matsui M, Yoshimori $\mathrm{T}$, Ohsumi Y. In vivo analysis of autophagy in response to nutrient starvation using transgenic mice expressing a fluorescent autophagosome marker. Mol Biol Cell. 2004;15(3):1101-1111.

10. Zhang L, et al. Small molecule regulators of autophagy identified by an image-based highthroughput screen. Proc Natl Acad Sci U S A 2007;104(48):19023-19028.

11. Liu J, et al. Beclin1 controls the levels of p53 by regulating the deubiquitination activity of USP10 and USP13. Cell. 2011;147(1):223-234. 
12. Shaw SY, et al. Selective modulation of autophagy, innate immunity, and adaptive immunity by small molecules. ACS Chem Biol. 2013;8(12):2724-2733.

13. Saitoh $\mathrm{T}$, et al. Loss of the autophagy protein Atg16L1 enhances endotoxin-induced IL-1 $\beta$ production. Nature. 2008;456(7219):264-268.

14. Larsen KB, Lamark T, Øvervatn A, Harneshaug I, Johansen T, Bjørkøy G. A reporter cell system to monitor autophagy based on p62/SQSTM1. Autophagy. 2010;6(6):784-793.

15. Williams A, et al. Novel targets for Huntington's disease in an mTOR-independent autophagy pathway. Nat Chem Biol. 2008;4(5):295-305.

16. Hosokawa N, et al. Nutrient-dependent mTORC1 association with the ULK1-Atg13-FIP200 complex required for autophagy. Mol Biol Cell. 2009;20(7):1981-1991.

17. Benjamin D, Colombi M, Moroni C, Hall MN. Rapamycin passes the torch: a new generation of mTOR inhibitors. Nat Rev Drug Discov 2011;10(11):868-880.

18. Vézina C, Kudelski A, Sehgal SN. Rapamycin (AY-22,989), a new antifungal antibiotic. I. Taxonomy of the producing streptomycete and isolation of the active principle. J Antibiot (Tokyo). 1975;28(10):721-726.

19. Rini BI. Temsirolimus, an inhibitor of mammalian target of rapamycin. Clin Cancer Res. 2008;14(5):1286-1290.

20. Gabardi S, Baroletti SA. Everolimus: a proliferation signal inhibitor with clinical applications in organ transplantation, oncology, and cardiology. Pharmacotherapy. 2010;30(10):1044-1056.

21. Mita M, Sankhala K, Abdel-Karim I, Mita A, Giles F. Deforolimus (AP23573) a novel mTOR inhibitor in clinical development. Expert Opin Investig Drugs. 2008;17(12):1947-1954.

22. Liu Q, Thoreen C, Wang J, Sabatini D, Gray NS. mTOR mediated anti-cancer drug discovery. Drug Discov Today Ther Strateg. 2009;6(2):47-55.

23. Harris H, Rubinsztein DC. Control of autophagy as a therapy for neurodegenerative disease. $\mathrm{Nat}$ Rev Neurol. 2012;8(2):108-117.

24. Chong ZZ, Shang YC, Wang S, Maiese K. Shedding new light on neurodegenerative diseases through the mammalian target of rapamycin. Prog Neurobiol. 2012;99(2):128-148.

25. Ravikumar B, et al. Inhibition of mTOR induces autophagy and reduces toxicity of polyglutamine expansions in fly and mouse models of Huntington disease. Nat Genet. 2004;36(6):585-595.

26. Apsel B, et al. Targeted polypharmacology: discovery of dual inhibitors of tyrosine and phosphoinositide kinases. Nat Chem Biol. 2008;4(11):691-699.

27. Thoreen CC, et al. An ATP-competitive mammalian target of rapamycin inhibitor reveals rapamycin-resistant functions of mTORC1. J Biol Chem. 2009;284(12):8023-8032.

28. Hsieh AC, et al. Genetic dissection of the oncogenic mTOR pathway reveals druggable addiction to translational control via $4 \mathrm{EBP}-\mathrm{eIF} 4 \mathrm{E}$. Cancer Cell. 2010;17(3):249-261.

29. Nyfeler B, et al. Relieving autophagy and 4EBP1 from rapamycin resistance. $\mathrm{Mol}$ Cell Biol. 2011;31(14):2867-2876.

30. White E, DiPaola RS. The double-edged sword of autophagy modulation in cancer. Clin Cancer Res. 2009;15(17):5308-5316.

31. Alers S, Löffler AS, Wesselborg S, Stork B. Role of AMPK-mTOR-Ulk1/2 in the regulation of autophagy: cross talk, shortcuts, and feedbacks. Mol Cell Biol. 2012;32(1):2-11.

32. Shaw RJ, et al. The kinase LKB1 mediates glucose homeostasis in liver and therapeutic effects of metformin. Science. 2005;310(5754):1642-1646.

33. Hardie DG. Neither LKB1 nor AMPK are the direct targets of metformin. Gastroenterology. 2006;131(3):973.

34. Liang XH, et al. Protection against fatal Sindbis virus encephalitis by beclin, a novel Bcl-2-interacting protein. J Virol. 1998;72(11):8586-8596.

35. Pattingre S, et al. Bcl-2 antiapoptotic proteins inhibit Beclin 1-dependent autophagy. Cell. 2005;122(6):927-939.

36. Shimizu S, et al. Role of Bcl-2 family proteins in a non-apoptotic programmed cell death dependent on autophagy genes. Nat Cell Biol. 2004;6(12):1221-1228.

37. Lipinski MM, et al. A genome-wide siRNA screen reveals multiple mTORC1 independent signaling pathways regulating autophagy under normal nutritional conditions. Dev Cell. 2010;18(6):1041-1052.

38. Miller S, et al. Shaping development of autophagy inhibitors with the structure of the lipid kinase Vps34. Science. 2010;327(5973):1638-1642.

39. Wu YT, et al. Dual role of 3-methyladenine in modulation of autophagy via different temporal patterns of inhibition on class I and III phosphoinositide 3-kinase. J Biol Chem. 2010;285(14):10850-10861.

40. Tolkovsky AM, Xue L, Fletcher GC, Borutaite V. Mitochondrial disappearance from cells: a clue to the role of autophagy in programmed cell death and disease? Biochimie. 2002;84(2-3):233-240.

41. Blommaart EF, Krause U, Schellens JP, VreelingSindelárová $\mathrm{H}$, Meijer AJ. The phosphatidylinositol 3-kinase inhibitors wortmannin and LY294002 inhibit autophagy in isolated rat hepatocytes. Eur J Biochem. 1997;243(1-2):240-246.

42. Xing C, Zhu B, Liu H, Yao H, Zhang L. Class I phosphatidylinositol 3-kinase inhibitor LY294002 activates autophagy and induces apoptosis through $\mathrm{p} 53$ pathway in gastric cancer cell line SGC7901. Acta Biochim Biophys Sin (Shanghai). 2008;40(3):194-201.

43. Itakura E, Kishi C, Inoue K, Mizushima N. Beclin 1 forms two distinct phosphatidylinositol 3-kinase complexes with mammalian Atg14 and UVRAG. Mol Biol Cell. 2008;19(12):5360-5372.

44. Correa RJ, et al. Combination of AKT inhibition with autophagy blockade effectively reduces ascites-derived ovarian cancer cell viability. Carcinogenesis. 2014;35(9):1951-1961.

45. Vakifahmetoglu-Norberg $\mathrm{H}$, et al. Chaperonemediated autophagy degrades mutant p53. Genes Dev. 2013;27(15):1718-1730.

46. Petiot A, Ogier-Denis E, Blommaart EF, Meijer AJ, Codogno P. Distinct classes of phosphatidylinositol 3'-kinases are involved in signaling pathways that control macroautophagy in HT-29 cells. J Biol Chem. 2000;275(2):992-998.

47. Ohwada J, et al. Discovery and biological activity of a novel class I PI3K inhibitor, CH5132799.
Bioorg Med Chem Lett. 2011;21(6):1767-1772.

48. Wallin JJ, et al. GDC-0980 is a novel class I PI3K/ mTOR kinase inhibitor with robust activity in cancer models driven by the PI3K pathway. Mol Cancer Ther. 2011;10(12):2426-2436.

49. Wallin JJ, et al. GDC-0941, a novel class I selective PI3K inhibitor, enhances the efficacy of docetaxel in human breast cancer models by increasing cell death in vitro and in vivo. Clin Cancer Res. 2012;18(14):3901-3911.

50. Hideshima T, et al. Perifosine, an oral bioactive novel alkylphospholipid, inhibits Akt and induces in vitro and in vivo cytotoxicity in human multiple myeloma cells. Blood. 2006;107(10):4053-4062.

51. Wei Y, et al. EGFR-mediated Beclin 1 phosphorylation in autophagy suppression, tumor progression, and tumor chemoresistance. Cell. 2013;154(6):1269-1284.

52. Wang RC, et al. Akt-mediated regulation of autophagy and tumorigenesis through Beclin 1 phosphorylation. Science. 2012;338(6109):956-959.

53. Gordon PB, Holen I, Fosse M, Rotnes JS, Seglen PO. Dependence of hepatocytic autophagy on intracellularly sequestered calcium. J Biol Chem. 1993;268(35):26107-26112.

54. Xia HG, et al. Control of basal autophagy by calpain1 mediated cleavage of ATG5. Autophagy. 2010;6(1):61-66.

55. Greenwood AF, Jope RS. Brain G-protein proteolysis by calpain: enhancement by lithium. Brain Res. 1994;636(2):320-326.

56. Ganley IG, Wong PM, Gammoh N, Jiang X. Distinct autophagosomal-lysosomal fusion mechanism revealed by thapsigargin-induced autophagy arrest. Mol Cell. 2011;42(6):731-743.

57. Carmichael J, Sugars KL, Bao YP, Rubinsztein DC. Glycogen synthase kinase-3beta inhibitors prevent cellular polyglutamine toxicity caused by the Huntington's disease mutation. J Biol Chem. 2002;277(37):33791-33798.

58. Sarkar S, et al. Lithium induces autophagy by inhibiting inositol monophosphatase. J Cell Biol. 2005;170(7):1101-1111.

59. Vicencio JM, et al. The inositol 1,4,5-trisphosphate receptor regulates autophagy through its interaction with Beclin 1. Cell Death Differ. 2009;16(7):1006-1017.

60. Criollo A, et al. Regulation of autophagy by the inositol trisphosphate receptor. Cell Death Differ. 2007;14(5):1029-1039.

61. Hochfeld WE, Lee S, Rubinsztein DC. Therapeutic induction of autophagy to modulate neurodegenerative disease progression. Acta Pharmacol Sin. 2013;34(5):600-604.

62. Shoji-Kawata S, et al. Identification of a candidate therapeutic autophagy-inducing peptide. Nature. 2013;494(7436):201-206.

63. Liu Y, et al. Autosis is a $\mathrm{Na}^{+}, \mathrm{K}^{+}$-ATPase-regulated form of cell death triggered by autophagy-inducing peptides, starvation, and hypoxia-ischemia. Proc Natl Acad Sci U S A . 2013;110(51):20364-20371.

64. Hoyvik H, Gordon PB, Berg TO, Stromhaug PE, Seglen PO. Inhibition of autophagic-lysosomal delivery and autophagic lactolysis by asparagine. JCell Biol. 1991;113(6):1305-1312.

65. Aplin A, Jasionowski T, Tuttle DL, Lenk SE, Dunn 
WA Jr. Cytoskeletal elements are required for the formation and maturation of autophagic vacuoles. JCell Physiol. 1992;152(3):458-466.

66. Yu QC, Marzella L. Modification of lysosomal proteolysis in mouse liver with taxol. Am J Pathol. 1986;122(3):553-561.

67. Mackeh R, Perdiz D, Lorin S, Codogno P, Poüs C. Autophagy and microtubules - new story, old players. J Cell Sci. 2013;126(pt 5):1071-1080.

68. Furuya T, et al. Negative regulation of Vps34 by Cdk mediated phosphorylation. Mol Cell. 2010;38(4):500-511.

69. Veldhoen RA, et al. The chemotherapeutic agent paclitaxel inhibits autophagy through two distinct mechanisms that regulate apoptosis. Oncogene. 2013;32(6):736-746.

70. Kawaguchi Y, Kovacs JJ, McLaurin A, Vance JM, Ito A, Yao TP. The deacetylase HDAC6 regulates aggresome formation and cell viability in response to misfolded protein stress. Cell. 2003;115(6):727-738.

71. Yan J, et al. SQSTM1/p62 interacts with HDAC6 and regulates deacetylase activity. PLoS One. 2013;8(9):e76016.

72. Lee JY, et al. HDAC6 controls autophagosome maturation essential for ubiquitin-selective qualitycontrol autophagy. EMBO J.2010;29(5):969-980.

73. Stankov MV, et al. Histone deacetylase inhibitors induce apoptosis in myeloid leukemia by suppressing autophagy. Leukemia. 2014;28(3):577-588.

74. Robert T, et al. HDACs link the DNA damage response, processing of double-strand breaks and autophagy. Nature. 2011;471(7336):74-79.

75. Carew JS, et al. Targeting autophagy augments the anticancer activity of the histone deacetylase inhibitor SAHA to overcome Bcr-Abl-mediated drug resistance. Blood. 2007;110(1):313-322.

76. Gammoh N, Lam D, Puente C, Ganley I, Marks $\mathrm{PA}$, Jiang $\mathrm{X}$. Role of autophagy in histone deacetylase inhibitor-induced apoptotic and nonapoptotic cell death. Proc Natl Acad Sci U S A. 2012;109(17):6561-6565.

77. Schneider P, Korolenko TA, Busch U. A review of drug-induced lysosomal disorders of the liver in man and laboratory animals. Microsc Res Tech. 1997;36(4):253-275.

78. Gunja N, et al. Survival after massive hydroxychloroquine overdose. Anaesth Intensive Care. 2009;37(1):130-133.

79. Amaravadi RK, Winkler JD. Lys05: a new lysosomal autophagy inhibitor. Autophagy. 2012;8(9):1383-1384.

80. Juhász G. Interpretation of bafilomycin, $\mathrm{pH}$ neutralizing or protease inhibitor treatments in autophagic flux experiments: novel considerations. Autophagy. 2012;8(12):1875-1876.

81. Choi HS, Jeong EH, Lee TG, Kim SY, Kim HR, Kim CH. Autophagy inhibition with monensin enhances cell cycle arrest and apoptosis induced by mTOR or epidermal growth factor receptor inhibitors in lung cancer cells. Tuberc Respir Dis (Seoul). 2013;75(1):9-17.

82. Carew JS, et al. Lucanthone is a novel inhibitor of autophagy that induces cathepsin D-mediated apoptosis. J Biol Chem. 2011;286(8):6602-6613.

83. Wang Z, et al. Matrine, a novel autophagy inhibitor, blocks trafficking and the proteolytic activation of lysosomal proteases. Carcinogenesis. 2013;34(1):128-138.

84. Tresse E, et al. VCP/p97 is essential for matura- tion of ubiquitin-containing autophagosomes and this function is impaired by mutations that cause IBMPFD. Autophagy. 2010;6(2):217-227.

85. Sasazawa Y, et al. Xanthohumol impairs autophagosome maturation through direct inhibition of valosin-containing protein. ACS Chem Biol. 2012;7(5):892-900.

86. Chou TF, et al. Reversible inhibitor of p97, $\mathrm{DBeQ}$, impairs both ubiquitin-dependent and autophagic protein clearance pathways. Proc Natl Acad Sci U S A. 2011;108(12):4834-4839.

87. Yamamoto A, Tagawa Y, Yoshimori T, Moriyama Y, Masaki R, Tashiro Y. Bafilomycin A1 prevents maturation of autophagic vacuoles by inhibiting fusion between autophagosomes and lysosomes in rat hepatoma cell line, $\mathrm{H}$-4-II-E cells. Cell Struct Funct. 1998;23(1):33-42.

88. Shacka JJ, Klocke BJ, Roth KA. Autophagy, bafilomycin and cell death: the "a-B-cs" of plecomacrolide-induced neuroprotection. Autophagy. 2006;2(3):228-230.

89. Renna M, et al. Azithromycin blocks autophagy and may predispose cystic fibrosis patients to mycobacterial infection. JClin Invest. 2011;121(9):3554-3563.

90. Moriyasu Y, Inoue Y. Use of protease inhibitors for detecting autophagy in plants. Methods Enzymol. 2008;451:557-580.

91. Donohue E, et al. Inhibition of autophagosome formation by the benzoporphyrin derivative verteporfin. J Biol Chem. 2011;286(9):7290-7300.

92. Rossi M, et al. Desmethylclomipramine induces the accumulation of autophagy markers by blocking autophagic flux. J Cell Sci. 2009;122(pt 18):3330-3339. 